\title{
ESTIMATIVA DA ESPESSURA E VARIAÇÃO LITOLÓGICA DO GRUPO URUCUIA NA BACIA DO RIO DE ONDAS NO OESTE DA BAHIA, USANDO SONDAGEM ELÉTRICA VERTICAL
}

\author{
THICKNESS AND LITOLOGICAL VARIATION ESTIMATION OF THE URUCUIA GROUP IN \\ THE ONDAS RIVER BASIN IN THE WEST OF THE STATE OF BAHIA, USING VERTICAL \\ ELECTRICAL SOUNDING
}

\author{
Joana Angélica Guimarães da LUZ ${ }^{1}$; Leandro MOUTINHO ${ }^{2}$; Alteredo Oliveira CUTRIM ${ }^{3}$ \\ ${ }^{1}$ Universidade Federal do Sul Sul da Bahia - Reitoria. Rua Itabuna, s/n, Rod. Ilhéus - Vitória da Conquista, km 39, BR 415, \\ Ferradas, Itabuna-BA. E-mail: jasguimaraes7@gmail.com. \\ ${ }^{2}$ Universidade Federal do Oeste da Bahia, Reitoria, Centro das Ciências Exatas e das Tecnologias., Rua Professor José Seabra de Lemos, 316, Recanto \\ dos Pássaros, Barreiras, BA - Brasil. E-mail: leandro.moutinho@ufob.edu.br \\ ${ }^{3}$ Universidade Federal de Mato Grosso - FAGEO- Faculdade de Geociências. Av. Fernando Corrêa da Costa, 2367 - Bairro Boa \\ Esperança. Cuiabá, MT. E-mail: alteredoc@ hotmail.com
}

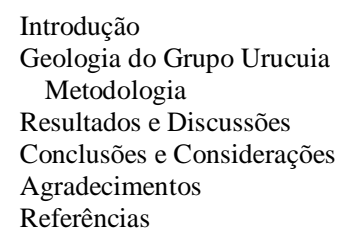

RESUMO - Esta pesquisa foi realizada no oeste do estado da Bahia, na bacia do Rio de Ondas, com o objetivo de estimar a espessura e a variação litológica do Grupo Urucuia, usando sondagem elétrica vertical (SEV). Foram realizadas oito SEV's, com abertura máxima de $\mathrm{AB} / 2$ de $800 \mathrm{~m}$ para investigar a profundidade máxima de $400 \mathrm{~m}$. Os resultados mostram que os solos têm espessura máxima de 2,94m e mínima de 0,90m e o Grupo Urucuia tem espessura em torno de 397m, divididos em arenito e arenito argiloso distribuídos em várias profundidades. Estas informações enriquecem o conhecimento geológico da região e são muito importantes para pesquisa hidrogeológica, envolvendo estimativa de reservas de água e avaliação da vulnerabilidade à contaminação do Urucuia, assim como para a elaboração de projetos de poços para explorar o sistema Aquífero Urucuia. Em geral estes resultados mostram também que o sistema Aquífero Urucuia é livre coberto, pois está sobreposto apenas por solo e rocha de elevada condutividade hidráulica.

Palavras-Chave: SEV, Bacia do rio de Ondas, Grupo Urucuia, Hidrogeologia.

\begin{abstract}
This research was carried out in the west of the state Bahia, in the Ondas river basin, Brazil, to estimate the thickness and lithological variation of the Urucuia Group using vertical electrical sounding (VES). Were realized eight SEV's with maximum opening of $800 \mathrm{~m}$ to investigate $400 \mathrm{~m}$ of depth. The results show that the soils have a maximum thickness of $2.94 \mathrm{~m}$ and a minimum thickness of $0.90 \mathrm{~m}$ and the Urucuia Group has a thickness around $397 \mathrm{~m}$, divided in sandstone and clayey sandstone distributed in several depths. This information enriches the geological knowledge of the region and is very important for hydrogeological research, involving estimation of water reserves and evaluation of vulnerability to contamination, as well as for the development of well projects to explore the Urucuia Aquifer system. These results also show that the Urucuia Aquifer system is free covered, because it is superimposed only by soil and rock of high hydraulic conductivity
\end{abstract}

Keywords: VES, Ondas river basin, Urucuia Group, Hydrogeology.

\section{INTRODUÇÃO}

A estimativa da espessura e profundidade de unidades geológicas de bacia sedimentar pode ser realizada através da técnica de sondagem elétrica vertical-SEV e perfil geológico de poços tubulares. Como em geral a profundidade de poço tubular está vinculada à demanda de água do seu proprietário, raramente um poço penetra toda espessura da bacia sedimentar, tornando parcial o conhecimento dessa informação. Assim, a aplicação de SEV integrada com dados de perfil geológico de poço tubular tem produzido excelentes resultados (Cutrim \& Shiraiwa, 2011; Cutrim et al., 2007; Cutrim \& Rebouças, 2005; Raimi et al., 2011; Coker, 2012; Okoro, 2010).
O conhecimento desses parâmetros é indispensável para viabilizar pesquisas hidrogeológicas envolvendo estimativa de reservas de aquífero, elaborar projeto de construção de poço tubular profundo (Costa, 2000; Cutrim \& Rebouças, 2005; Custódio \& Llamas, 1976), e para avaliar a vulnerabilidade à contaminação de aquíferos (Foster et al., 2002; Cutrim \& Campos, 2010a,b; Barbosa, et al., 2007).

No oeste do estado da Bahia, na bacia do Rio de Ondas (Figura 1), o conhecimento da profundidade e espessura do Grupo Urucuia está limitado aos perfis geológicos de poços tubulares, cuja profundidade está vinculada à 
demanda de água do dono do poço, tornando assim um conhecimento parcial desses parâmetros. Desse modo, este trabalho tem como objetivo estimar esses parâmetros nessa bacia hidrográfica, usando a técnica de sondagem elétrica vertical (SEV) para viabilizar pesquisas hidrogeológicas e melhorar o conhecimento geológico dessa bacia.

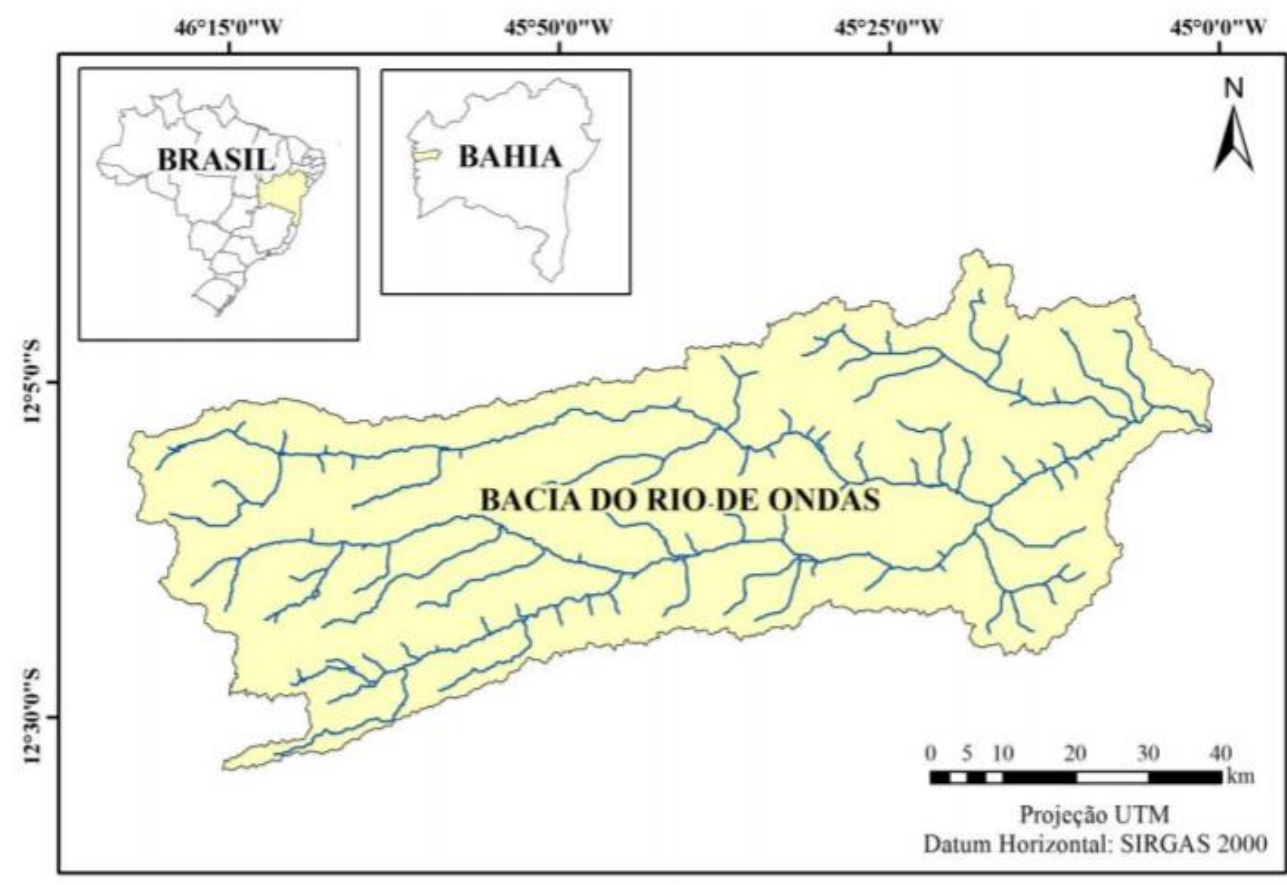

Figura 1 - Localização da bacia do rio de Ondas (Fistaro, et al.,2015 ).

\section{GEOLOGIA DO GRUPO URUCUIA}

O Grupo Urucuia (GU), de idade neo-cretácea e de espessura máxima de $400 \mathrm{~m}$, é composto pelas formações Posse e Serra das Araras, depositadas em ambientes eólico e fluvial, com contato erosivo abrupto entre elas (Spigolon \& Alvarenga, 2002). A Formação Posse é a base do GU, ocorre sobreposta às rochas do Grupo Bambuí, é constituída por arenitos finos a médios, bem selecionados, com estratificação cruzada de grande porte, ocasionalmente silicificados em porções no contato com a Formação Serra das Araras. A Formação Serra das Araras é composta por conglomerados, arenito e siltito de espessura máxima de $2 \mathrm{~m}$. Perfis geológicos de poços de monitoramento instalados na bacia (Figura 2 e Quadro 1) mostram detalhamento da litologia, porém a profundidade máxima dos poços é de $103 \mathrm{~m}$.

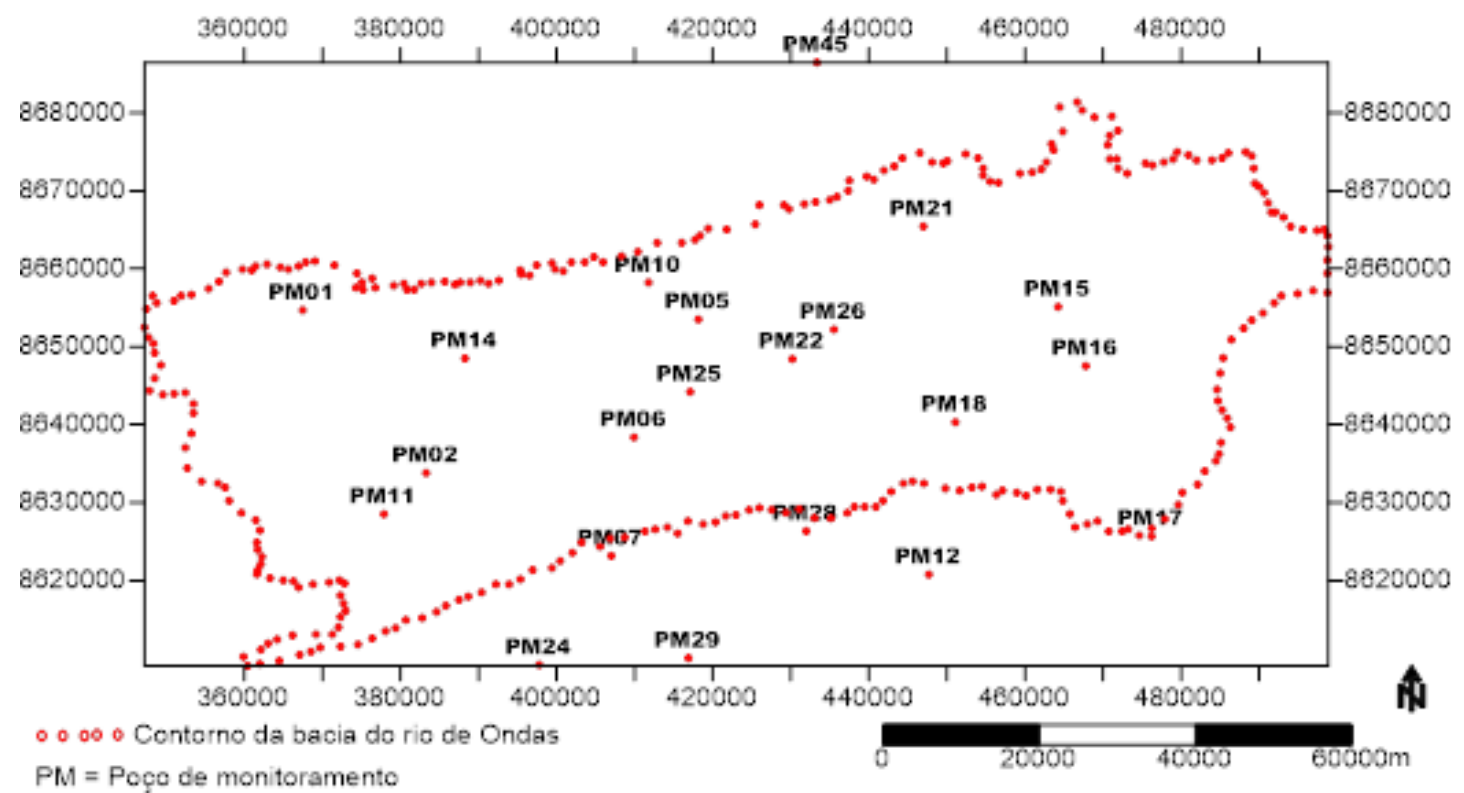

Figura 2 - Distribuição dos poços de monitoramento na bacia do Rio de Ondas. 
Quadro 1 - Perfil geológico de poços de monitoramento na bacia do Rio de Ondas.

\begin{tabular}{|c|c|c|c|c|c|c|c|}
\hline \multirow[t]{2}{*}{ Poço } & \multicolumn{2}{|c|}{$\begin{array}{l}\text { Coorde } \\
\text { nadas UTM }\end{array}$} & \multirow{2}{*}{$\begin{array}{l}\text { Solo areno- } \\
\text { argiloso } \\
\text { - Prof (m) }\end{array}$} & \multirow[t]{2}{*}{$\begin{array}{l}\text { Conglomerado } \\
\text {-Prof }(\mathrm{m})\end{array}$} & \multirow[t]{2}{*}{$\begin{array}{l}\text { Arenito fino a } \\
\text { médio -Prof (m) }\end{array}$} & \multirow{2}{*}{$\begin{array}{l}\text { Arenito } \\
\text { argiloso - } \\
\text { Prof(m) }\end{array}$} & \multirow{2}{*}{\begin{tabular}{|l} 
Arenito \\
silicificado \\
-Prof(m)
\end{tabular}} \\
\hline & $\mathrm{X}$ & $\bar{Y}$ & & & & & \\
\hline PM01 & 367476 & 8654685 & $0 \times 1$ & $\cdots$ & 1.93 & - & $\cdots$ \\
\hline PM02 & 383291 & 8633806 & $0 \times 1$ & 27 a 32 & 1 a $27 ; 32$ a 103 & $-\ldots$ & $\ldots$ \\
\hline PM05 & 418166 & 8653516 & $0 \times 2$ & 2 a $6 ; 12$ a 20 & 6 a $12 ; 20$ a 64 & $-\cdots$ & $\ldots$ \\
\hline PM06 & 409932 & 8638379 & $0 \times 8$ & 8 a $10 ; 48$ a $50 ; 60$ a 61 & 10 a $48 ; 50$ a 6061 a 62 & $-\infty$ & $\cdots$ \\
\hline PM10 & 411775 & 8658229 & $0 \times 2$ & -...- & $2 \mathrm{a} 71,5$ & $-\ldots$ & $\ldots$ \\
\hline PM11 & 377893 & 8628522 & $0 \times 2$ & - & 2 a 110 & $-\cdots$ & $\ldots$ \\
\hline PM25 & 417108 & 8644202 & $0 \times 2$ & $\ldots$ & 2 a 114 & $-\cdots$ & $\ldots$ \\
\hline PM07 & 407015 & 8623158 & $0 \times 1$ & 9 a $13 ; 22$ a 23 & 1 a 9,13 a 62 & -+- & $\cdots$ \\
\hline PM17 & 476223 & 8625662 & 0.2 & $\cdots$ & 11 a 82 & $2 \mathrm{a} 11$ & $\cdots$ \\
\hline PM12 & 447689 & 8620777 & $0 \times 1$ & $\ldots$ & $1 \mathrm{a} 72$ & $-\ldots$ & $-\ldots$ \\
\hline PM24 & 397771 & 8609184 & $0 \times 2$ & $\ldots$ & $2 \times 77$ & $-\ldots$ & $77 \mathrm{a} 92$ \\
\hline PM28 & 431980 & 8626334 & 0 a 18 & 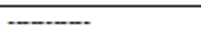 & $\cdots$ & 18 a 64 & -ב- \\
\hline PM29 & 416900 & 8610045 & $0 \times 2$ & 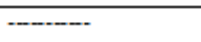 & 18 a 74 & 2 a 14 & 14 a 18 \\
\hline PM14 & 388265 & 8648503 & $0 \times 22$ & $\ldots$ & 46 a 48 & 22 a 46 & $48 \mathrm{a} 71$ \\
\hline PM15 & 464249 & 8655113 & $0 \times 2$ & 26 a 30 e 36 a 42 & 2 a $26 ; 30$ a $36 ; 42$ a 102 & $\cdots$ & - \\
\hline PM18 & 451098 & 8640289 & 0,8 & 10 a 18 e 22 a 30 & 8 a $10 ; 18$ a 22 e 30 a 51 & -+- & $\cdots$ \\
\hline PM16 & 467794 & 8647530 & $\ldots$ & $-\overline{-}$ & 0 a $23 ; 25$ a 68 & 23 a $25 ; 68$ a 72 & 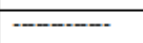 \\
\hline PMD1 & 446994 & 8665448 & $0 \times 1$ & 48 a 52 & 1 a $48 ; 56$ a 82 & 52 a 56 & $\ldots+\ldots$ \\
\hline PM22 & 430205 & 8648417 & $0 \mathrm{a} 18$ & $+\cdots$ & $\cdots$ & 18 a 32 & $32 \mathrm{a} 60$ \\
\hline PM26 & 435546 & 8652208 & $0 \mathrm{a} 14$ & $\cdots$ & $20 \times 51$ & 14 a 20 & $\cdots$ \\
\hline PM45 & 433353 & 8686484 & $0 \times 2$ & $18 \mathrm{a} 25 ; 66 \mathrm{a} 72$ & 2 a $18 ; 64$ a $66 ; 78$ a 80 & 25 a $30 ; 72$ a 78 & $30 \mathrm{a} 64$ \\
\hline
\end{tabular}

\section{METODOLOGIA}

A técnica de Sondagem Elétrica Vertical SEV é uma ferramenta eficiente na definição de contato litológico contendo bom contraste de resistividade, como é comum em meio geológico acamadado (Cutrim \& Rebouças, 2005; Amorim Júnior \& Lima, 2007; Asfahani, 2007; Cutrim et al., 2007; Okoro, 2010; Cutrim \& Shiraiwa, 2011; Raimi et al, 2011; Atzemoglou \& Tsourlos,
2012; Coker, 2012; Cutrim \& Dias, 2014).

A técnica consiste em injetar corrente elétrica no solo em dois pontos e medir o potencial em dois outros pontos. A resistividade aparente é obtida a partir da diferença de potencial e da corrente que circulou em subsuperfície e do fator geométrico, através da equação apresentada em Bhattacharya \& Patra (1986):

$$
\boldsymbol{\rho}_{\mathrm{a}}=\left(\frac{\Delta \mathbf{V}}{\mathrm{I}}\right) \mathbf{k}, \quad k=2 \pi\left(\frac{1}{A M}-\frac{1}{B M}-\frac{1}{A N}+\frac{1}{B N}\right)
$$

Onde: $\rho_{\mathrm{a}}=$ resistividade aparente; $\Delta \mathrm{v}=$ diferença de potencial; $\mathrm{I}=$ corrente elétrica; $\mathrm{AM}=$ distância entre os eletrodos $\mathrm{A}$ e $\mathrm{M}$; $\mathrm{AN}=$ distância entre os eletrodos $\mathrm{A}$ e $\mathrm{N} ; \mathrm{BM}=$ distância entre os eletrodos $\mathrm{B}$ e $\mathrm{M}$ e $\mathrm{BN}=$ distância entre os eletrodos $\mathrm{B}$ e N.

$\mathrm{Na}$ coleta dos dados em campo foi utilizado o arranjo Schlumberger (Figura 3), cuja resistividade aparente é dada pela equação:

$$
\rho_{a}=\pi\left(a^{2}-b^{2}\right) \cdot \frac{\Delta V}{I}, \operatorname{com} A B / 2=a \text { e } M N / 2=b .
$$

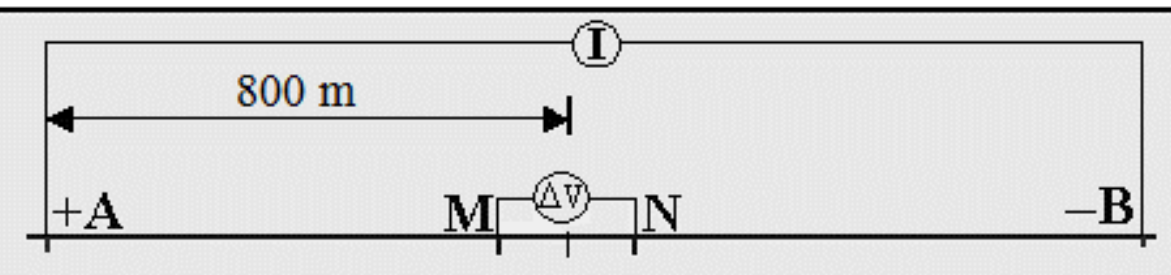

Figura 3 - Arranjo Schlumberger. 


\section{RESULTADOS E DISCUSSÕES}

Foram realizadas oito $\mathrm{SEVs}$ com abertura máxima de $\mathrm{AB} / 2$ de $800 \mathrm{~m}$, cuja distribuição na área está na Figura 4. Os resultados dessas SEVs estão apresentados no Quadro 2 e nas Figuras 5 e
6. O número de camadas do perfil geoelétrico de cada SEV não representa o número de unidades geológicas, mas sim o número de camadas que permite o melhor ajuste da curva da SEV.

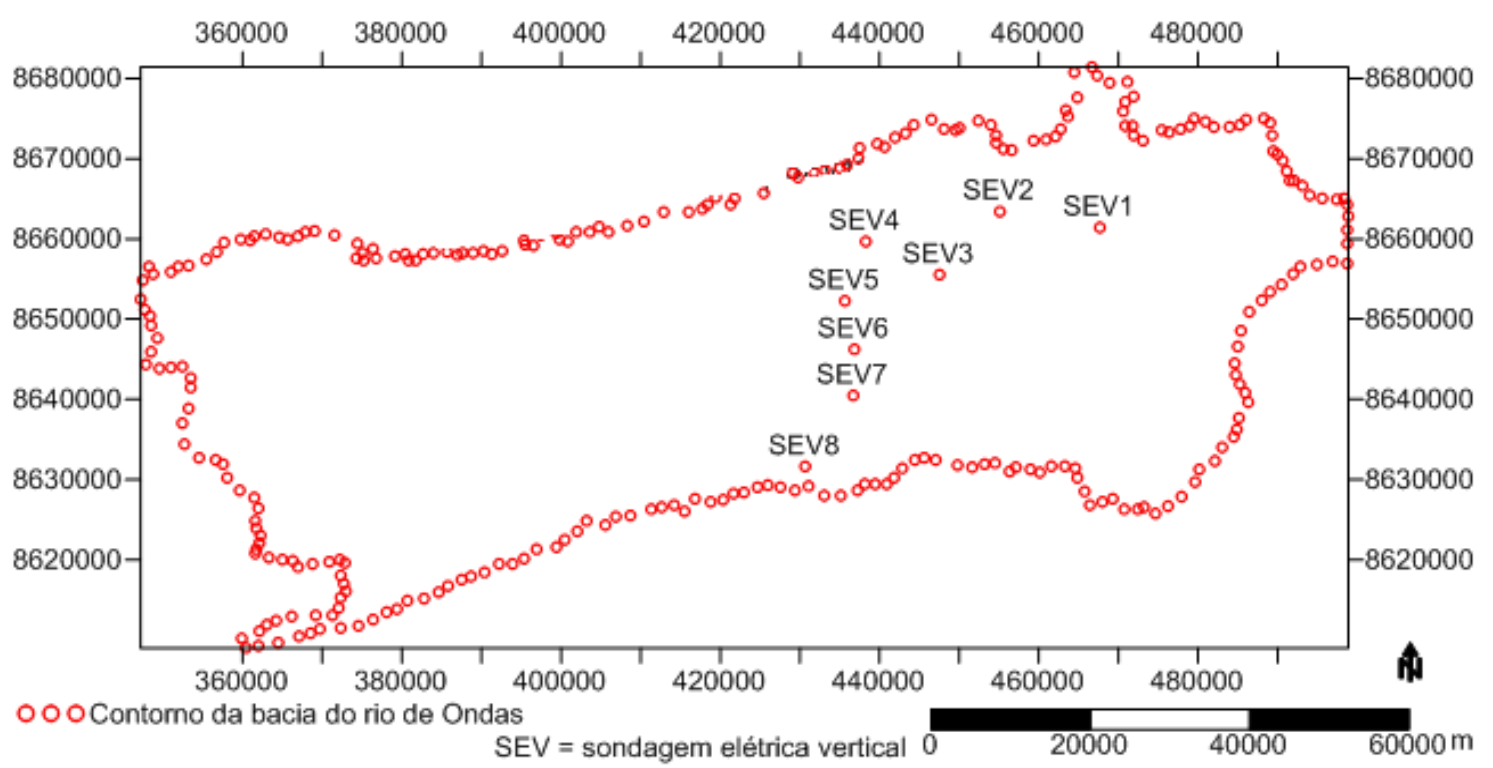

Figura 4 - Localização das SEVs na área de estudo.

A SEV1 foi ajustada usando um modelo de seis camadas. Esta SEV permitiu estimar uma camada de solo de $1,93 \mathrm{~m}$ de espessura e três camadas que constituem o Grupo Urucuia, sendo uma camada de arenito argiloso de $3,21 \mathrm{~m}$ espessura, uma camada de arenito de $118 \mathrm{~m}$ e uma de arenito argiloso de 276,86m.

A SEV2 foi ajustada usando um modelo de dez camadas. As duas primeiras correspondem ao solo com espessura de $1,08 \mathrm{~m}(\mathrm{AB} / 2 \pm 2 \mathrm{~m})$; as seis camadas seguintes $(A B / 2 \pm 178 \mathrm{~m})$ são de arenito com espessura de $89,42 \mathrm{~m}$; a nona camada $(\mathrm{AB} / 2 \pm 420 \mathrm{~m})$ tem espessura de $209 \mathrm{~m}$, correspondendo a um arenito argiloso, e a decima $(\mathrm{AB} / 2 \pm 200 \mathrm{~m})$ é arenito com espessura de $100 \mathrm{~m}$, corresponde ao substrato da SEV.

A SEV3 foi ajustada usando um modelo de seis camadas. As três primeiras camadas $(\mathrm{AB} / 2$ $\pm 3,60 \mathrm{~m}$ ) são de solo de espessura de $1,8 \mathrm{~m}$, a quarta camada $(\mathrm{AB} / 2 \pm 112 \mathrm{~m})$ é arenito de $56 \mathrm{~m}$ de espessura, a quinta camada $(\mathrm{AB} / 2 \pm 266 \mathrm{~m})$ é arenito argiloso com espessura de $33 \mathrm{~m}$ e a sexta camada $(\mathrm{AB} / 2 \pm 418 \mathrm{~m})$ é arenito com espessura de $209 \mathrm{~m}$, corresponde ao substrato da SEV.

A SEV4 foi ajustada usando um modelo de oito camadas. As três primeiras camadas $(\mathrm{AB} / 2$ $\pm 4,40 \mathrm{~m}$ ) correspondem a solo de espessura de $2,19 \mathrm{~m}$, as três camadas seguintes $(\mathrm{AB} / 2 \pm 293 \mathrm{~m})$ são de arenito de 146,8m de espessura, a sétima camada $(\mathrm{AB} / 2 \pm 292 \mathrm{~m})$ é arenito argiloso com espessura de $146 \mathrm{~m}$ e a oitava camada, o substrato da SEV $(\mathrm{AB} / 2 \pm 210 \mathrm{~m})$, é arenito com espessura de $105 \mathrm{~m}$.

A SEV5 foi ajustada com um modelo de seis camadas. As duas primeiras camadas $(\mathrm{AB} / 2$ $\pm 1,80 \mathrm{~m}$ ) são correspondentes a solo de espessura de $0,9 \mathrm{~m}$, a terceira camada $(\mathrm{AB} / 2 \pm 11,6 \mathrm{~m})$ é arenito argiloso com $5,82 \mathrm{~m}$ de espessura, a quarta e a quinta camadas $(\mathrm{AB} / 2 \pm 202 \mathrm{~m})$ correspondem a arenito com espessura de 101,3 $m$ e a sexta camada o substrato da SEV (AB/2 $\pm 584 \mathrm{~m})$ é arenito argiloso com espessura de 292,09 m.

A SEV6 foi ajustada usando um modelo de sete camadas. As duas primeiras camadas $(\mathrm{AB} / 2$ $\pm 5,20 \mathrm{~m}$ ) são de solo com espessura de $2,64 \mathrm{~m}$, as quatro camadas seguintes com $(\mathrm{AB} / 2 \pm 482,9$ m) são de arenito com $241,46 \mathrm{~m}$ de espessura, e a sétima camada, com $(A B / 2 \pm 312 \mathrm{~m})$, é arenito argiloso com espessura de $155,9 \mathrm{~m}$ e representa o substrato da SEV.

A SEV7 foi ajustada usando um modelo de sete camadas. As duas primeiras camadas $(\mathrm{AB} / 2 \pm 5,40 \mathrm{~m})$ correspondem a solo com espessura de 2,94 m, as três camadas seguintes $(\mathrm{AB} / 2 \pm 186 \mathrm{~m})$ são de arenito com 92,96 m de espessura, a sexta camada com $(\mathrm{AB} / 2 \pm 346 \mathrm{~m})$ 
é arenito argiloso com espessura de $173 \mathrm{~m} \mathrm{e}$ a sétima camada, com $(\mathrm{AB} / 2 \pm 346 \mathrm{~m})$, é um arenito de espessura de 131, $1 \mathrm{~m}$ é o substrato da SEV.

A SEV8 foi ajustada usando um modelo de seis camadas. As duas primeiras camadas
$(\mathrm{AB} / 2 \pm 3,0 \mathrm{~m})$ são correspondentes ao solo de espessura de $1,5 \mathrm{~m}$, as três camadas seguintes com $(\mathrm{AB} / 2 \pm 347 \mathrm{~m})$ são de arenito com 173,5 $m$ de espessura, e a sexta camada, que marca o substrato da $\operatorname{SEV}(\mathrm{AB} / 2 \pm 450 \mathrm{~m})$, é arenito argiloso com espessura de $225 \mathrm{~m}$.

Quadro 2 - Perfil geológico da Grupo Urucuia gerado pelas SEVs.

\begin{tabular}{|c|c|c|c|c|c|c|c|c|c|}
\hline \multirow[t]{3}{*}{ SEV } & & \multicolumn{8}{|c|}{ Espessura e profundidade de litológico do Grupo Urucuia gerado pelas SEVs } \\
\hline & \multirow{2}{*}{$\begin{array}{l}\text { Solo } \\
\mathrm{h}(\mathrm{m})\end{array}$} & \multicolumn{2}{|c|}{ Arenito } & \multicolumn{2}{|c|}{ Arenito } & \multicolumn{2}{|c|}{ Arenito } & \multicolumn{2}{|c|}{ Arenito argiloso } \\
\hline & & $\mathbf{h}(\mathbf{m})$ & $\mathrm{d}(\mathrm{m})$ & $\mathbf{h}(\mathbf{m})$ & $\mathrm{d}(\mathrm{m})$ & $\mathbf{h}(\mathbf{m})$ & $\mathrm{d}(\mathrm{m})$ & $\mathbf{h}(\mathbf{m})$ & $\mathbf{d}(\mathbf{m})$ \\
\hline 1 & 1,93 & $-\cdots$ & $\ldots$ & 3,21 & 1,93 & 118 & 5,13 & 276,86 & 123,13 \\
\hline 2 & 1,08 & 89,42 & 1,08 & 209 & 90,6 & 100 & 300 & -..-- & $-\cdots$ \\
\hline 3 & 1,84 & 56 & 1,84 & 133 & 57,8 & 209 & 191 & $\ldots$ & $-\cdots$ \\
\hline 4 & 2,19 & 146,81 & 2,19 & 146 & 149 & 105 & 295 & -.--. & $-\cdots$ \\
\hline 5 & 0,90 & $-\cdots$ & $\ldots$ & 5,82 & 0,90 & 101,3 & 6,73 & 292,09 & 107,91 \\
\hline 6 & 2,64 & 241,46 & 2,64 & 155,9 & 244 & -.-- & $-\cdots$ & $\ldots$ & $\cdots$ \\
\hline 7 & 2,94 & 92,96 & 2,94 & 173 & 95,9 & 131,1 & 269 & $\ldots$ & $\ldots$ \\
\hline 8 & 1.5 & 173.5 & 1,5 & 225 & 175 & --.-- & - & $-\cdots$ & \\
\hline
\end{tabular}

$\mathrm{h}=$ espessura $\mathrm{d}=$ profundidade.

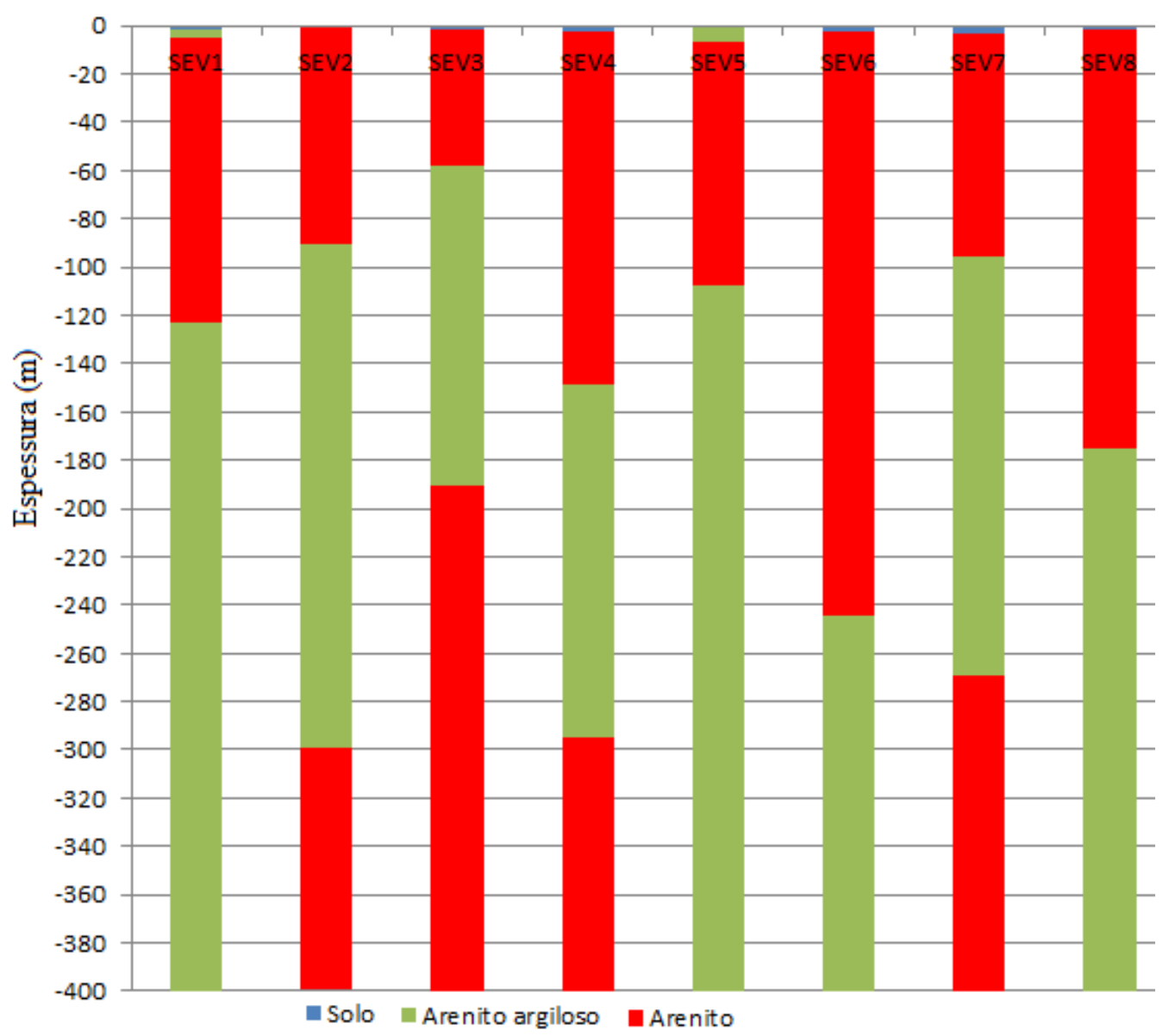

Figura 5 - Modelo geológico do Grupo Urucuia produzido pelas SEVs.

Ressalta-se que as curvas das SEVs 1 e 5 apresentam conformação similar para todas as $\mathrm{AB} / 2$, porém os valores de resistividade são maiores na SEV1. No entanto, a indicação de maior e contínua zona saturada ocorre nas duas SEVs a partir de $\mathrm{AB} / 2$ de $150 \mathrm{~m}$, onde a resistividade começa a decrescer. As curvas das SEVs 2 e 3 têm forma semelhante, porém a resistividade da SEV2 é muito maior que aquela da SEV3, no entanto a indicação de maior e contínua zona saturada ocorre nas duas SEVs a partir de $\mathrm{AB} / 2$ de $50 \mathrm{~m}$, onde a resistividade começa a decrescer. 


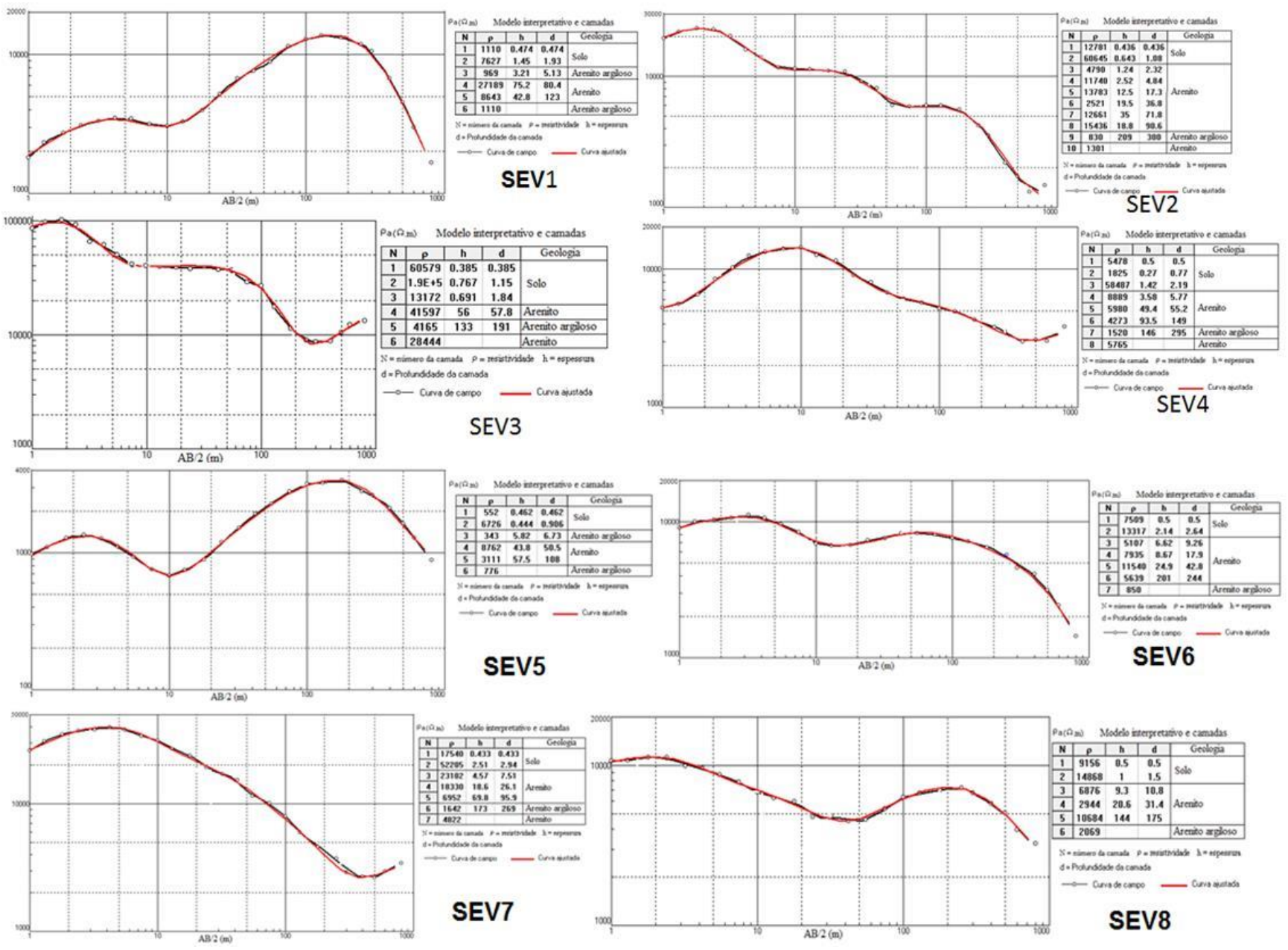

Figura 6 - Perfis geoelétricos das SEVs e modelos interpretados.

As SEVs 4 e 7 tem conformação semelhante, a resistividade começa a decrescer a partir de $\mathrm{AB} / 2$ de $10 \mathrm{~m}$, indicando a profundidade de ocorrência da maior e contínua zona saturada. As SEVs 6 e 8 apresentam conformação similar, porém a indicação de maior e contínua zona saturada ocorre na SEV6 a partir de AB/2 de $50 \mathrm{~m}$ e na SEV8 a partir de AB/2 de 200m, onde a resistividade começa a decrescer.

Essa variação marcante da resistividade nas SEVs e entre elas está relacionada, principalmente, à variação litológica do Grupo Urucuia e, em segundo lugar, à presença de água.

\section{CONCLUSÕES E CONSIDERAÇÕES}

A sondagem elétrica vertical-SEV foi eficiente para estimar a profundidade, espessura e variação litológica do Grupo Urucuia, porém não foi possível definir as suas formações geológicas. Foram estimadas camadas de arenito e arenito argiloso com espessura e profundidade variadas.

Nas SEVs 1, 5, 6 e 8 ocorrem o arenito e o arenito argiloso com maior espessura na sequência, sendo que o arenito tem maior espessura na SEV6, nas demais SEVs tem-se a sequência do arenito, arenito argiloso e arenito. Observa-se que ao longo do perfil o arenito tem a profundidade máxima de $7 \mathrm{~m}$ na SEV5 e a maior espessura na SEV6, no centro da bacia. De modo geral esses resultados indicam também que o sistema Aquífero Urucuia é livre coberto, pois está sobreposto apenas por solo e rocha de elevada condutividade hidráulica.

Estes dados enriquecem o conhecimento geológico da região e podem subsidiar pesquisas hidrogeológicas, envolvendo construção de poço tubular profundo, quantificação de reservas de águas e avaliação de vulnerabilidade à contaminação desse sistema Aquífero. 


\section{AGRADECIMENTOS}

Os autores agradecem à FINEP (Financiadora de Estudos e Projetos do Ministério da Ciência e Tecnologia, Chamada Pública MCT/Finep CT Hidro 01/2010) pela concessão do apoio financeiro à pesquisa.

\section{REFERENCIAS}

AMORIM JÚNIOR, V. \& LIMA, O.A.L. 2007. Avaliação hidrogeológica do aquífero Urucuia na bacia do rio das Fêmeas usando resistividade e polarização elétrica induzida. Revista Brasileira de Geofísica. 25(2): 117-129. 2007.

ASFAHANI, J. Geoelectrical investigation for characterizing the hydrogeological conditions in semi-arid region in Khanasser valley, Syria. Journal of Arid Environments 68, 31-52. 2007.

ATZEMOGLOU, A.; TSOURLOS, P. 2D interpretation of vertical electrical soundings: application to the Sarantaporon basin (Thessaly, Greece). Journal of Geophysics and Engineering 9, 50-59. 2012.

BARBOSA, D. V., ZIMMER, J. R., CUTRIM, A. O., RUIZ, A. S. Avaliação da vulnerabilidade à contaminação do Aquífero Furnas na área urbana de Rondonópolis (MT), utilizando o método AVI. $1^{\circ}$ Simpósio de Recursos Hídricos do Norte e Centro Oeste Sul. Cuiabá-MT. 2007. (CD-ROM).

BHATTACHARYA, P.K. \& PATRA, H.P. Direct Current Geoelectric Sounding. Elsevier Publishing Company, New York, 134p. 1986.

COKER, J.O. 2012. Vertical electrical sounding (VES) methods to delineate potential groundwater aquifers in Akobo area, Ibadan, South-western, Nigeria. Journal of Geology and Mining Research Vol. 4(2), p. 35-42.

COSTA W.D. Uso e gestão de água subterrânea. In: Feitosa F. A. C., Manoel Filho J. (eds.). Hidrogeologia conceitos e aplicações. Fortaleza: CPRM. P.341-365. 2000.

CUSTODIO, E. \& LLAMAS, M. R. Hidrologia subterrânea. Tomo II, Ediciones Omega, S.A, Barcelona. p.1165-2359. 1976.

CUTRIM, A.O. \& REBOUÇAS, A.C. Estimativa das reservas de água do Aquífero Furnas na cidade de Rondonópolis-MT. In: I Simpósio de Recursos Hídricos do Sul - I Simpósio de Águas da AUGM, 2005, Santa Maria -RS. I AGUASUL, 2005. v. (CD Rom)

CUTRIM, A.O. \& REBOUÇAS, A.C. Aplicação de sondagem elétrica vertical na estimativa do topo e da espessura de unidade geológicas da Bacia do Paraná na cidade de Rondonópolis-MT. Revista Brasileira de Geofísica, v. 23, n. 1, p. 89-98. 2005.

CUTRIM, A.O. \& SHIRAIWA, S. Prospecção de água subterrânea no sudoeste do município de Rondonópolis (MT) usando sondagem elétrica vertical. Revista Brasileira de Geofísica, v. 29, n. 4, p. 45 - 51. 2011.

CUTRIM, A.O. \& CAMPOS, J.E.G. Aplicação dos métodos Drastic e Posh para a determinação da vulnerabilidade e perigo à contaminação do Aquífero Furnas na cidade de Rondonópolis-MT. Revista Brasileira de Recursos Hídricos, vol.15, n2, p.127-142. 2010a.
CUTRIM, A.O.; CAMPOS, J.E.G. Avaliação da vulnerabilidade e perigo à contaminação do Aquífero Furnas na cidade de Rondonópolis (MT) com aplicação dos métodos God e Posh. Revista Geociências UNESP Rio Claro (SP), v.29 (3): p. 401411. $2010 \mathrm{~b}$.

CUTRIM, A.O.; DIAS, F.S. Estimativa de profundidade e espessura de unidades geológicas da bacia do Paraná, usando sondagem elétrica vertical, no município de Poxoréo (MT). Revista Geociências, UNESP, v. 33, n.3, p.393-402. 2014.

CUTRIM, A.O.; RUIZ, A.S.; LIPORONI, L.M.; MEDEIROS, F.A.; BARROSO, U.C.; NASCIMENTO A.L. Sondagem elétrica vertical aplicada em pesquisa hidrogeológica na Bacia do Parecis, MT. Revista Brasileira de Geofísica, v. 25, n. 2, p.131-140. 2007.

FISTAROL, P.H.B.; BRANDOLFF, R.S.; SANTOS, J.Y.G. Análise Fisiográfica da Bacia do Rio de Ondas - BA. Anais XVII Simpósio Brasileiro de Sensoriamento Remoto - SBSR, João Pessoa-PB, Brasil, 25 a 29 de abril de 2015, INPE. CD

FOSTER, S.; HIRATA, R.; GOMES, D.; D’ELIA, M.; PARIS, $M$. Groundwater quality protection: a guide for water service companies, municipal authorities and environment agencies. World Bank, GWMATE. Washington, 101p. 2002.

OKORO, E.I; EGBOKA, B.C.E; ONWUEMESI, A.G. Evaluation of the aquifer characteristic of Nanka Sands using hydrogeological method in combination with Vertical Electrical Sounding (VES). J. Appl. Sci. Environ. Manage. v. 14, n. 2, p. 5 - 9. 2010.

RAIMI, J.; ABDULKARIM, M.S.; HAMIDU; ARABI, A. S. 2011. Application of Schlumberger Vertical Electrical Sounding for Determination of Suitable Sites for Construction of Boreholes for Irrigation Scheme within a Basement Complex. International Journal of Multidisciplinary Sciences and Engineering, v. 2, no. 6, p. 81-84.

SPIGOLON A.L.D.; ALVARENGA C.J.S. de. Fáceis e elementos arquiteturais resultantes de mudanças climáticas em um ambiente desértico: Grupo Urucuia (Neocretáceo), Bacia Sanfranciscana. Revista Brasileira de Geociências, 32(4): 579-586. 2002.

TIKHONOV, A.N. \& ARSENIN, V.Y. 1977. Solutions of illposed problems. Winston \& Sons, New York, 349p.

Submetido em 18 de agosto de 2018 Aceito em 22 de janeiro de 2020 